\title{
The Impact of Sunlight Intensity and Outdoor Temperature on the Performance of Inorganic Solar Panels
}

\author{
Haval M. Abdulla ${ }^{a}$, Fahmi F. Muhammad ${ }^{b, *}$ and Mohammad G. Faraj \\ Soft Materials \& Devices Lab, Department of Physics, Faculty of Science \& Health, \\ Koya University, Koya, Kurdistan Region, Iraq. \\ ahaval.muhammed@koyauniversity.org, bfahmi.fariq@koyauniversity.org, \\ cmohammad.ghaffar@koyauniversity.org
}

Keywords: inorganic solar panels, sunlight intensity, ambient temperature, I-V characteristic, $P-V$ characteristic, fill factor.

\begin{abstract}
In this work the impact of sunlight intensity and ambient temperature on the inorganic solar panels in winter climate (22 November 2015) at Sarwchawa, Kurdistan Region, Iraq was carried out. A maximum intensity of sunlight (106.25 klux) was reached at 12:00 PM. However, beyond 12:00 PM the intensity showed a non-monotonic change behavior. This was ascribed to the change of sun position, its orientation and the direction at which the surface of the panel is illuminated. Results showed that the increase of sunlight intensity has made $I_{s c}$ to increase, while that of the $V_{o c}$ remains relatively unchanged. The reason why $I_{s c}$ is more affected by the sunlight intensity was understood from the fact that charge carriers acquire enough kinetic energy to move towards their corresponding electrodes before they relapse or recombine together, while as $V_{o c}$ is mostly correlated to the energy gap. There was a trivial decrement of efficiency with the increase of temperature. This was where the $F F$ showed a reverse trend of increment with the increase of temperature. Noteworthy, at the points where the fill factor was decreased the efficiency was increased and vice versa.
\end{abstract}

\section{Introduction}

Solar energy is the most promising renewable power source because of its free usage, clean, eco-friendly and silent operation when it is utilized to generate electricity by means of solar cell (photovoltaic) panels $[1,2]$. The majority of today's solar panels available in the market are made from inorganic semiconductor materials, of them approximately $85 \%$ are based on crystalline silicon [3]. Silicon can offer many advantages like high crystal quality, stability, non-pollutant, and ideal band gap $\left(E_{g}=1.11 \mathrm{eV}\right)$ for solar energy absorption [4]. The photovoltaic cells use P-N junctions [5] to directly convert sunlight into electricity through the photovoltaic effect. The solar cells performance can be affected by the change in light intensity and temperature of the environment. This is theoretically interpreted as the response of the absorbent layer in solar cells is changed in terms of charge carrier generation and recombination [6] when it is subjected to the variation of light intensity and temperature. Light intensity is changed daily due to the rotation of the earth around its own axis, and seasonally due to the rotation of the earth around the sun [7]. Solar panels are made from regularly arranged arrays of solar cells interconnected together according to the required voltage and current. Consequently, it is crucial to understand the real performance of solar panels for outdoor applications under various environmental conditions. Without having enough information regarding the panels behavior one cannot effectively size and design the photovoltaic systems. Intensive literature review reveals that in order to understand the performance of solar cells under variable light intensity and temperature, researchers have utilized solar simulators, and/or filtered lamp intensities in the laboratories (indoor condition) [7-13]. However, little attention has been paid on the practical investigation of inorganic solar panels under different sunlight intensities and temperatures in outdoor situations. Therefore, in this research work we investigate the impact of sunlight intensity and ambient temperature on the electrical performance of inorganic solar panels based on silicon. The solar panels are installed in an outdoor condition in Sarwchawa town, located in Kurdistan Region, Iraq. 


\section{Methodology}

Two small silicon based solar panels (Leybold Didactic GMBH) with dimension of $25 \times 25 \mathrm{~cm}^{2}$ connected in series were installed on the roof of a home building specified for the investigations. The panels were inclined 36 degrees due south and a light meter was used to measure the light intensity, accordingly. Two digital multimeters were utilized to measure the current and voltage produced by the panels, while a variable resistor ( $\Omega$ to $100 \mathrm{k} \Omega$ ) was connected as a load and a thermometer was used to measure the ambient temperature. Figure 1 shows the experimental set up used in the investigations.

The maximum power $\left(P_{\max }\right)$ delivered from the solar panels and the fill factor $(F F)$ were calculated by the following equations:

$P_{\text {max }}=V_{m p} \times I_{m p}$

$F F=P_{\max } /\left(V_{o c} \times I_{s c}\right)$

Where, $I_{s c}$ and $V_{o c}$ are short-circuit current and open-circuit voltage of the solar panels, respectively.

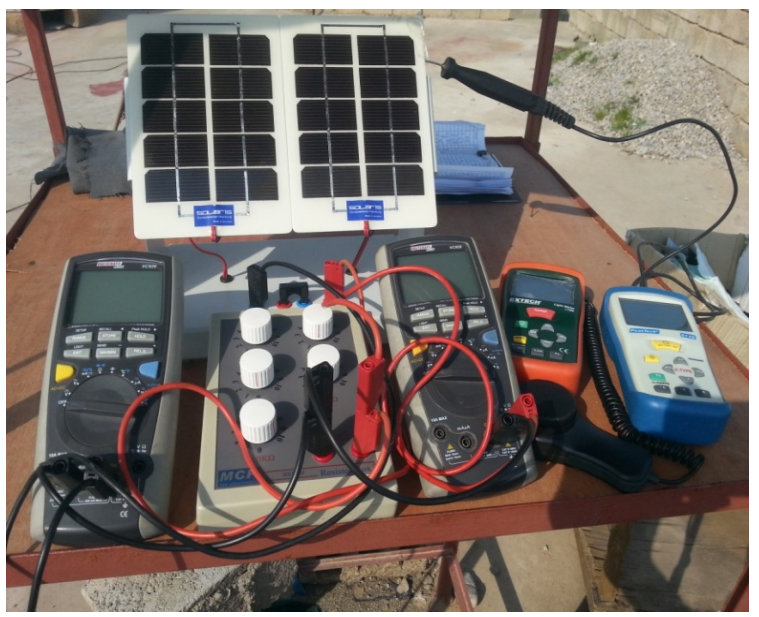

Figure 1: The experimental setup to measure $I-V$ and $P-V$ of the panels in outdoor condition.

\section{Results and discussion}

Figure 2 shows the sunlight intensity versus time from 7:00 AM to 4:00 PM recorded on the day 22 November 2015. One can notice that the intensity is increased with the preceding of time, such that a maximum intensity of sunlight (106.25 klux) can be reached at 12:00 PM. However, beyond 12:00 PM the intensity showed a stepwise decrement, making the intensity spectra to show a non-monotonic change behavior. This can be ascribed to the change of sun position, its orientation and the direction at which the surface of the panel is illuminated [14]. The change in sunlight intensity (insolation) can be directly employed to predict the quality of electrical power generated by the panels.

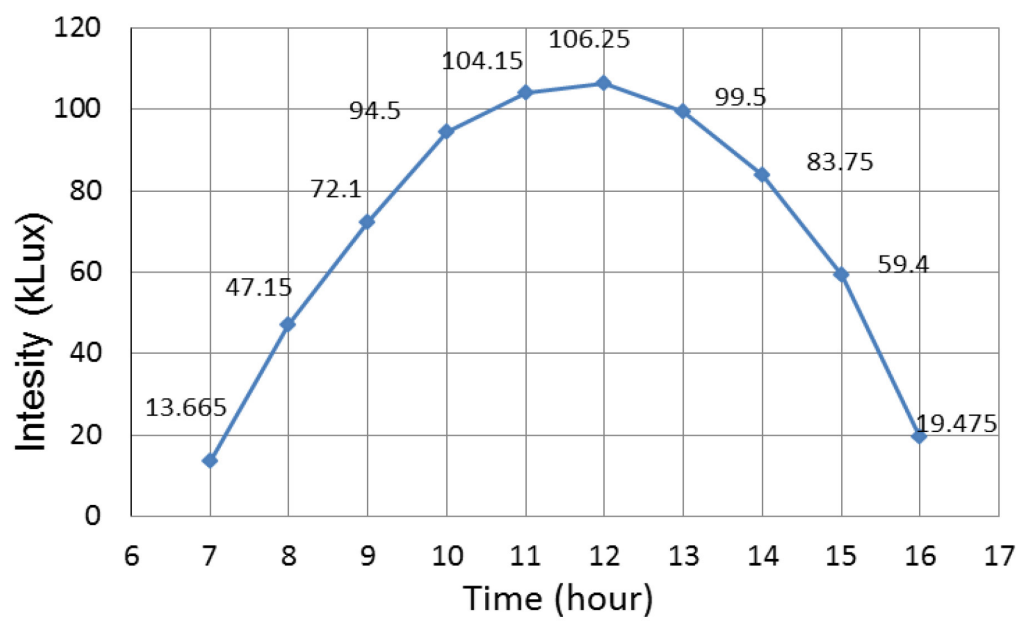

Figure 2: The intensity of sunlight versus time from 7:00 AM to 4:00 PM. 
Figure 3 shows the current-voltage $(I-V)$ characteristics of the solar panels under investigation at different times of the day (22 November 2015). Noteworthy, the current generated by the solar panels was increased by the preceding of time from 7:00 AM to about 12:00 PM. In support to our previous discussion, the increment of sunlight intensity has acted upon increasing the photo-electron generations [15] and hence a larger amount of current can be delivered to the output load. On contrary, beyond the noon time, the current generation was seen to decrease fairly because of the decrement in sunlight intensity. These evidences can be clearly proved from Figure 3 that the curve deviation shape along y-axis is more pronounced in comparison to that of the $\mathrm{x}$-axis. The shape consistency of all the curves at various irradiation times defines the inherent property of bilayer solar cells, where a knee position on each curve is definitely presented [16]. This $I-V$ curve behavior results in the production of a maximum power point $\left(P_{\max }\right)$, at which the external load is exactly matched with the internal resistance of the solar panel [17] .

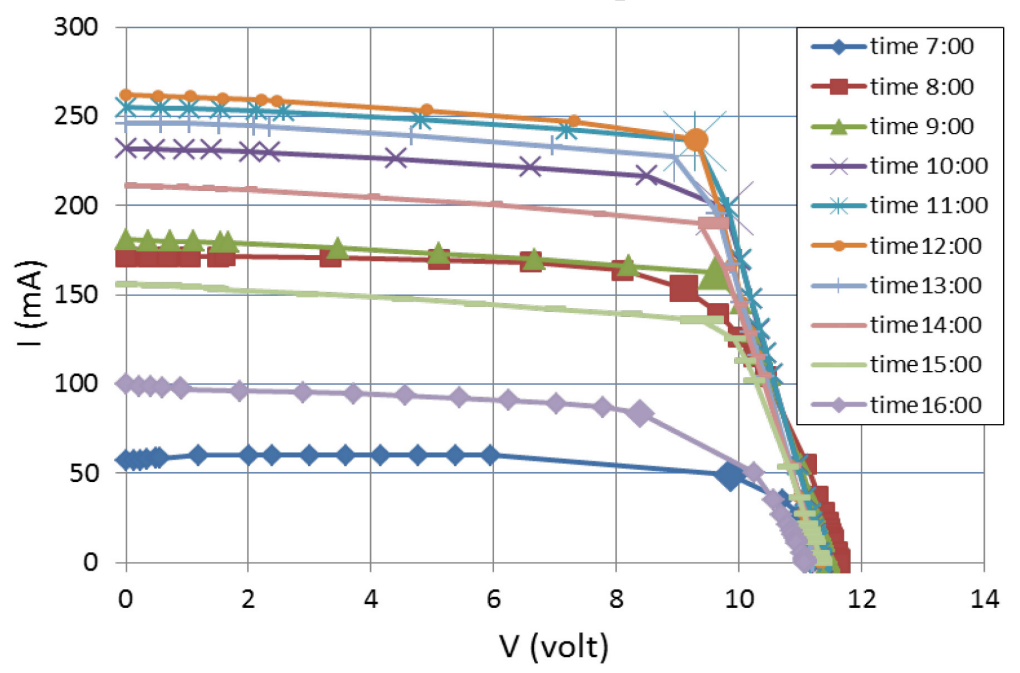

Figure 3: $I-V$ characteristics of solar panels in the time range from 7:00 AM to 4:00 PM.

Figure 4 shows the power-voltage $(P-V)$ characteristics curve for the solar panels under investigation at various times of the day. It was noticed that the maximum power $\left(P_{\max }\right)$ point is shifting up (increased) by the proceeding of time from early morning to the mid-noon (7:00 AM to 12:00 PM). This was attributed to the increased sunlight intensity that illuminating the surface of the solar panels (see Figure 2). The variation in $P_{\max }$ with time is depicted in Figure 5, indicating that the highest reachable power for the solar panels is considered to be at the mid-noon (12:00 PM) and it was counted to be about $2.3 \mathrm{~W}$. It is worth mentioning that the increment in the maximum power is not regular and there are some deviations compared to the regular change of the time and/or the solar insolation (in terms of intensity). This can be referred to the secondary impact of ambient temperature acting upon the performance of the solar panels, which will be discussed later.

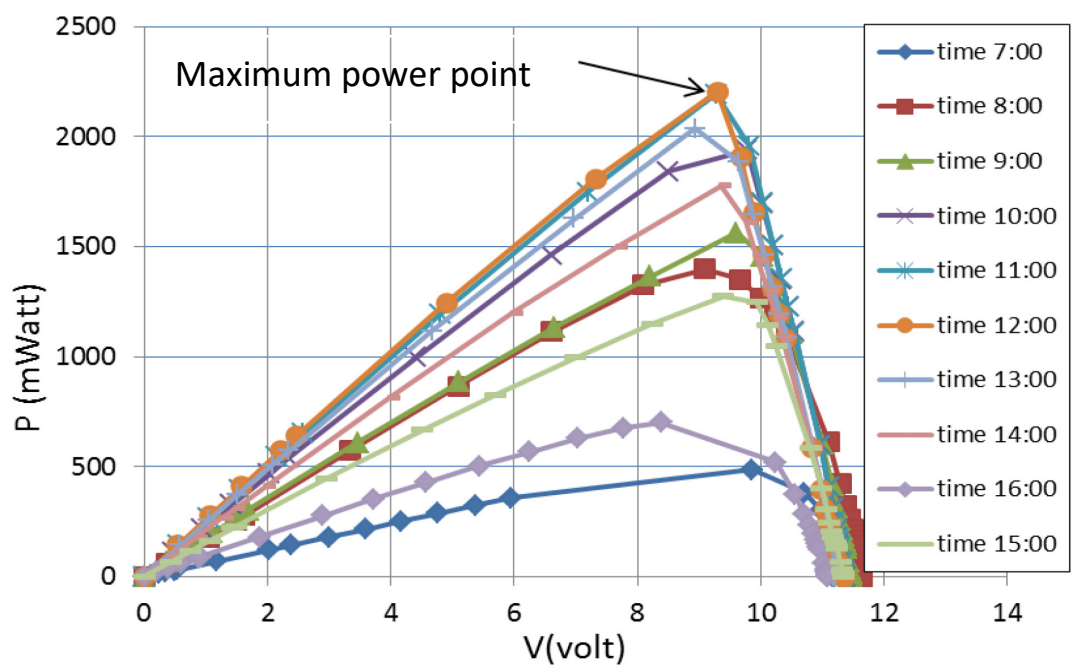

Figure 4: $P-V$ characteristics of solar panels in the time range from 7:00 AM to 4:00 PM. 


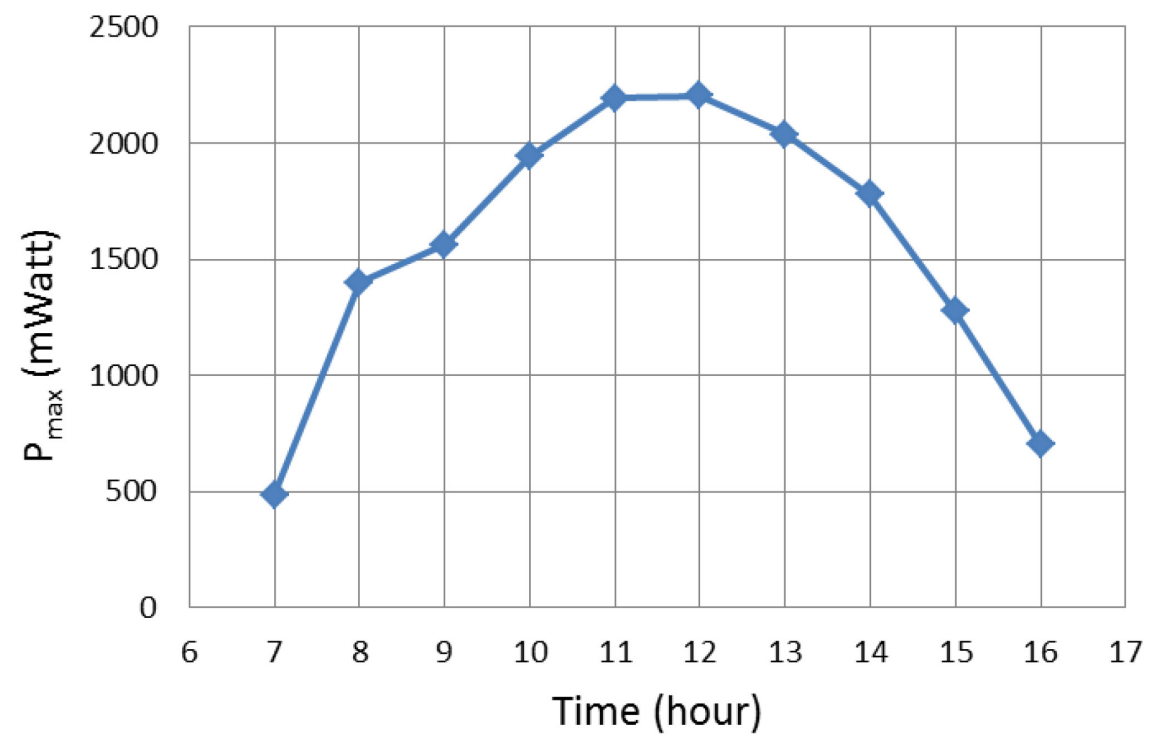

Figure 5: $P_{\max }$ versus time from 7:00 AM to 4:00 PM.

In order to elaborate on the effect of sunlight intensity on the important electrical parameters of solar panels, namely open circuit voltage $\left(V_{o c}\right)$ and short circuit current $\left(I_{s c}\right)$, Figure 6 was plotted. It was found in Figure 6 that the impact of sunlight intensity on $I_{s c}$ is towards the increment of its value, while that of the $V_{o c}$ remains relatively unchanged. The reason why $I_{s c}$ is more affected by the sunlight intensity can be understood from the fact that charge carriers (free electrons and holes) acquire enough kinetic energy to move towards their corresponding electrodes effectively before they relapse or recombine together. Nevertheless, $V_{o c}$ is directly correlated to the energy gap of the p-n junction active layer, which is very less affected by the variation of sunlight intensity. Instead, the increase in temperature can affect the band gap [18] but this was not obviously seen in our results because of the relatively low ambient temperature and good stability of the silicon based solar panels.

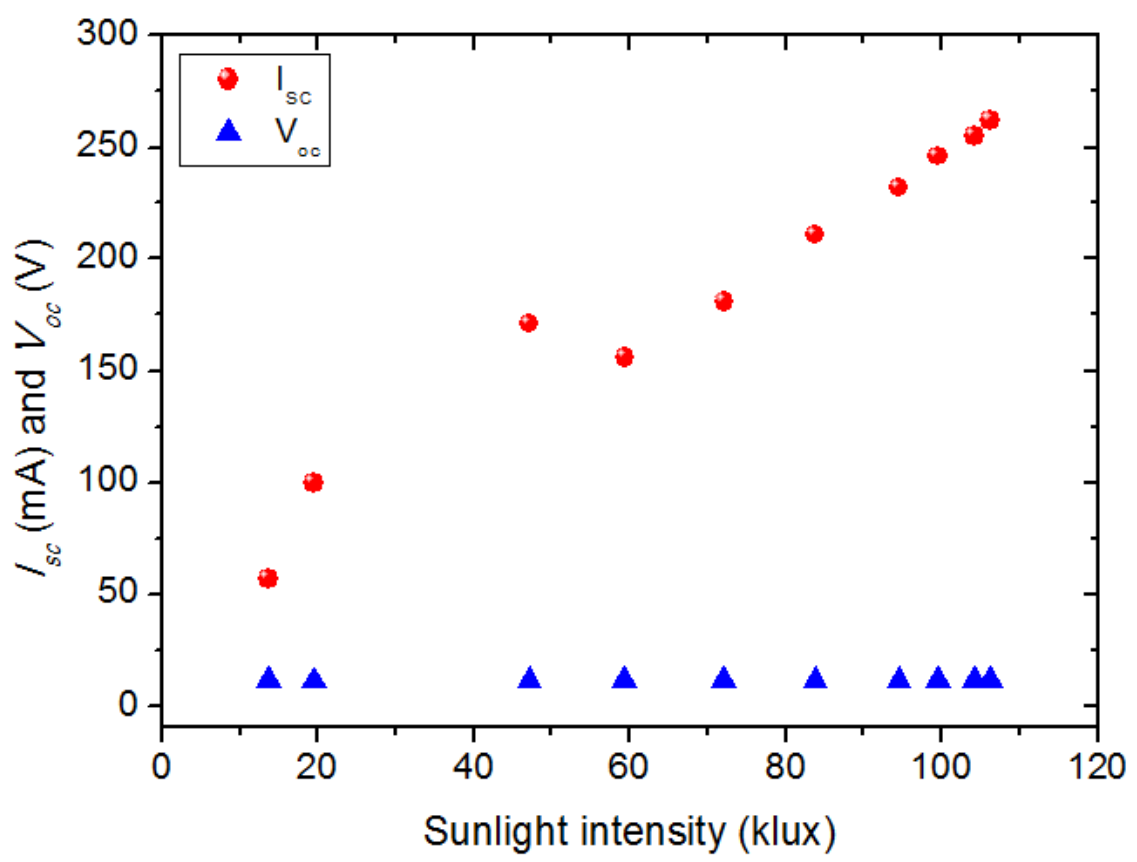

Figure 6: Open circuit voltage and short circuit current versus sunlight intensity for the inorganic solar panels. 
Temperature is another factor affecting the performance and output power of the solar panels. Figure 7 shows the ambient temperature change with respect to the proceeding time from early morning (7:00 AM) to early evening (4:00 PM). Unstable temperature change in the early morning was noticed due to the impact of wind, cloud, and position of the sun.

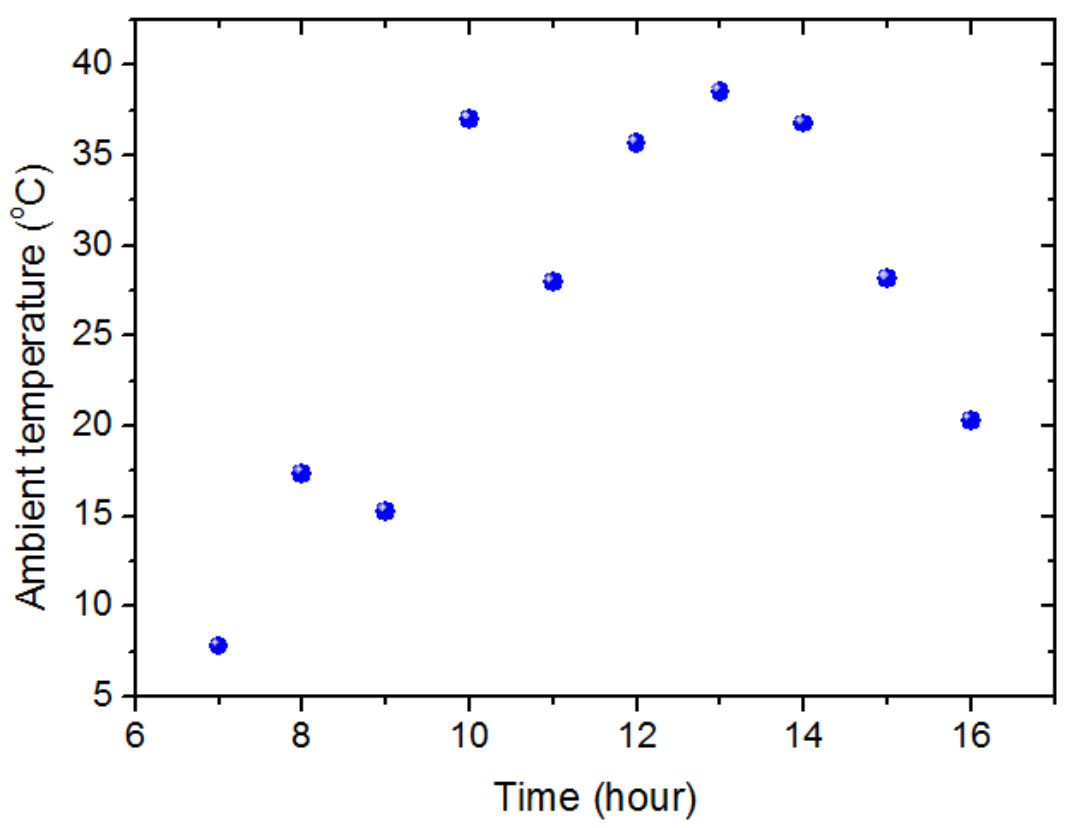

Figure 7: Ambient temperature versus time from 7:00 AM to 4:00 PM.

Fill factor $(F F)$ is another parameter approving the solar panels to show a non-linear electrical nature. Fill factor is also an indicator of the quality of solar panels. The higher fill factor value, the better solar panel performance. Figure 8 shows the change in $F F$ and efficiency $(\eta)$ with the change of ambient temperature. Noticeably, both parameters are not very much affected by the ambient temperature range from 7 to $39{ }^{\circ} \mathrm{C}$. However, upon close inspection into the figure, one can say that there is a relatively decrement trend of efficiency with the increase of temperature. This is where the $F F$ showed a reverse trend of increment with the increase of temperature. Further studies on the solar panels in different seasons may elucidate the exact changes directions. What is important to notice here is that at the points where the fill factor is decreased the efficiency is increased and vice versa (see the vertical dashed lines drawn in Figure 8).

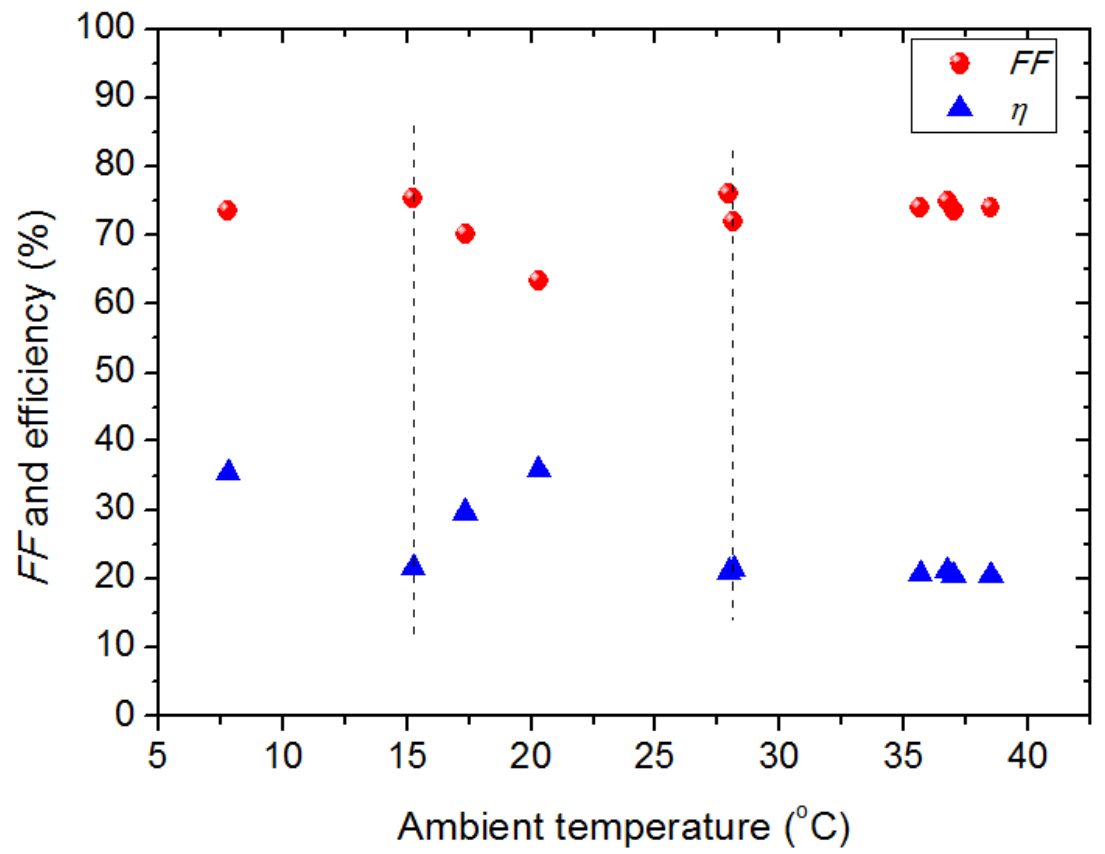

Figure 8: Fill factor and efficiency versus ambient temperature for inorganic solar panels. 


\section{Conclusion}

Investigation on the impact of sunlight intensity and ambient temperature on the inorganic solar panels in winter climate (22 November 2015) at Sarwchawa, Kurdistan Region, Iraq was carried out. One can notice that the intensity is increased with the preceding of time, such that a maximum intensity of sunlight (106.25 klux) can be reached at 12:00 PM. However, beyond 12:00 PM the intensity showed a stepwise decrement, making the intensity spectra to show a nonmonotonic change behavior. This can be ascribed to the change of sun position, its orientation and the direction at which the surface of the panel is illuminated. Noteworthy, the increment of sunlight intensity has acted upon increasing the photo-electron generations and hence a larger amount of current can be delivered to the output load. The highest reachable power for the solar panels was considered to be at the mid-noon (12:00 PM) and it was counted to be about $2.3 \mathrm{~W}$. The impact of sunlight intensity on $I_{s c}$ was to increase its value, while that of the $V_{o c}$ remains relatively unchanged. The reason why $I_{s c}$ is more affected by the sunlight intensity can be understood from the fact that charge carriers (free electrons and holes) acquire enough kinetic energy to move towards their corresponding electrodes effectively before they relapse or recombine together. There was a relatively decrement trend of efficiency with the increase of temperature. This is where the $F F$ showed a reverse trend of increment with the increase of temperature. Noteworthy, at the points where the fill factor was decreased the efficiency was increased and vice versa.

\section{Acknowledgement}

The authors are thankful to the Department of Physics, Faculty of Science and Health, Koya University for providing the necessary experimental items and for the administrative supports offered to this research work.

\section{References}

[1] S.Z. Sdeeq, A.H. Ameen, F.F. Muhammad, Effect of Series and Parallel Shading on the Photovoltaic Performance of Silicon Based Solar Panels, Journal of Technology Innovations in Renewable Energy, 4 (2015) 152-156.

[2] S. Kumar, T. kaur, Solar PV Performance-Issues and Challenges, International journal of innovative research in electrical, electronics, instrumentation and control engineering, 2 (2014) $2168-2172$.

[3] J. Szlufcik, S. Sivoththaman, J.F. Nlis, R.P. Mertens, R.V. Overstraeten, Low-cost industrial technologies of crystalline silicon solar cells, Proceedings of the IEEE, 85 (1997) 711-730.

[4] D. Yu, M. Yin, L. Lu, H. Zhang, X. Chen, X. Zhu, J. Che, D. Li, Silicon Solar Cells: HighPerformance and Omnidirectional Thin-Film Amorphous Silicon Solar Cell Modules Achieved by 3D Geometry Design (Adv. Mater. 42/2015), Advanced Materials, 27 (2015) 6768-6768.

[5] D.P. Wurfel, Physics of solar cells, Wiley Vch, 2016.

[6] J. Kurpiers, D.M. Balazs, A. Paulke, S. Albrecht, I. Lange, L. Protesescu, M.V. Kovalenko, M.A. Loi, D. Neher, Free carrier generation and recombination in PbS quantum dot solar cells, Applied Physics Letters, 108 (2016) 103102.

[7] A. El-Shaer, M. Tadros, M. Khalifa, Effect of Light intensity and Temperature on Crystalline Silicon Solar Modules Parameters, in, Citeseer, 2014.

[8] P. Arjyadhara, A. S.M, J. Chitralekha, Analysis of Solar PV cell Performance with Changing Irradiance and Temperature, International Journal Of Engineering And Computer Science, 2 (2013) 214-220.

[9] X. Cai, S. Zeng, X. Li, J. Zhang, S. Lin, A. Lin, B. Zhang, Effect of light intensity and temperature on the performance of GaN-based p-i-n solar cells, in: Electrical and Control Engineering (ICECE), 2011 International Conference on, (2011) 1535-1537.

[10] A.A. Jadallah, D.Y. Mahmood, Z.A. Abdulqader, Modeling and Simulation of a Photovoltaic Module in Different Operating Regimes, Acta Physica Polonica A, 128 (2015) 461-464. 
[11] S.M. Salih, F.F. Salih, M.L. Hasan, M.Y. Bedaiawi, Performance Evaluation of Photovoltaic Models Based on a Solar Model Tester, I.J. Information Technology and Computer Science, 7 (2012) 1-10.

[12] M.S. Salim, J.M. Najim, S.M. Salih, Majid Shahatha Salim[a]; Jassim Mohammed Najim[b]; Salih Mohammed Salih[Energy Science and Technology, 6 (2013) 36-40.

[13] D.M. Tobnaghi, D. Naderi, The Effect of Solar Radiation and Temperature on Solar cells Performance, Extensive Journal of Applied Sciences, 3 (2015) 39-43.

[14] M.-F. Loutre, D. Paillard, F. Vimeux, E. Cortijo, Does mean annual insolation have the potential to change the climate?, Earth and Planetary Science Letters, 221 (2004) 1-14.

[15] F.F. Muhammad, Design approaches to improve organic solar cells, Journal of Technology Innovations in Renewable Energy, 3 (2014) 1-8.

[16] F.F. Muhammad, K. Sulaiman, Photovoltaic performance of organic solar cells based on DH6T/PCBM thin film active layers, Thin Solid Films, 519 (2011) 5230-5233.

[17] N. Femia, G. Petrone, G. Spagnuolo, M. Vitelli, Optimization of perturb and observe maximum power point tracking method, Power Electronics, IEEE Transactions on, 20 (2005) 963973.

[18] K. O'Donnell, X. Chen, Temperature dependence of semiconductor band gaps, Applied Physics Letters, 58 (1991) 2924-2926. 